\title{
Numerical Predictions of Heat Transfer Performance in Dimple/Protrusion Channels with the Working Fluid of Supercritical Carbon Dioxide at High Re
}

\author{
Qi Jing ${ }^{1, \text { a }}$, Qiuwan Du ${ }^{1, b}$, Li Xiong ${ }^{1, c}$ and Di Zhang ${ }^{1, d}$ \\ ${ }^{1}$ School of Energy and Power Engineering, Xi'an Jiaotong University, Xi'an, China \\ ajingqi@stu.xjtu.edu.cn, bduqiuwan@stu.xjtu.edu.cn, cwodejianlizhi@163.com, \\ dzhang_di@mail.xjtu.edu.cn
}

Keywords: Dimple; Protrusion; Supercritical carbon dioxide; Heat transfer

Abstract. The RANS method and realizable $k-\varepsilon$ turbulence model are adopted to numerically investigate the turbulent flow and heat transfer performance of dimple/protrusion arranged in rectangular channel with the working fluid of supercritical carbon dioxide and air. Investigated $R e$ is 90000. The $\mathrm{Nu}$ distribution, the flow resistance coefficient $f$ and the comprehensive thermal performance TP were obtained. According to the results, it is obtained that the $N u / N u_{0}$ of supercritical carbon dioxide cases is about 2.3 times higher than the values of air cases at the $R e$ of 90000. The $f / f_{0}$ of supercritical carbon dioxide cases is close to the values of air cases. The TP of supercritical carbon dioxide cases is about 2.4 times higher than the values of air cases. The conclusion can be obtained that supercritical carbon dioxide has significant heat transfer performance while not increasing the flow resistance at high $R e$. Furthermore, the supercritical carbon dioxide dimple case possesses the best comprehensive thermal performance following the supercritical carbon dioxide protrusion case.

\section{Introduction}

Dimple/protrusion structure has good heat transfer enhancement performance while producing less flow resistance than other passive control structure, so its comprehensive thermal performance is superior to other types of heat transfer augmentation devices. Many researchers pay more attention to dimple/protrusion structure in recent years.

The earliest studies on the dimple/protrusion are based on its outstanding performance in flow control. This kind of structure is applied to the golf ball surface to achieve the effect of flow drag reduction. Belen'kiy et al. [1] give the experiment data which shows the improvement in heat transfer and pressure losses remain at approximate levels with flat channels. Moon et al. [2] describe the thermal characteristics of rectangular channels arranged with dimples, and find that the heat transfer enhancement factor is maintained at about 2.1 while corresponding flow resistance coefficient is in the range of 1.6-2.0 in the fully developed flow conditions. Ligrani et al. [3] focus on the flow characteristics of channels arranged with dimples in one side, it is presented that channels arranged with protrusions in opposite wall own larger local friction factor and local $\mathrm{Nu}$ than channels without protrusions. Lan et al. [4] study on the heat transfer performance of the stationary micro-channels arranged with dimples. Shen et al. [5] give computational simulation results which show the flow and heat transfer performance of the U-shaped cooling channels arranged with dimples.

Currently, most of all papers related to the heat transfer of dimples/protrusions take air and water as the working fluid. Carbon dioxide has a low critical point and its supercritical condition possesses excellent properties in flow and heat transfer performance. The present paper deals with the heat transfer characteristics of conventional rectangular channels with dimples/protrusions placed on bottom and takes supercritical carbon dioxide as the working fluid. The $\mathrm{Nu}$ distribution, the flow resistance coefficient $f$ and the comprehensive thermal performance TP were obtained. Comparing with the heat transfer factors of air conditions, the heat transfer characteristics of supercritical carbon dioxide were obtained. 


\section{Physical Model and Numerical Method}

Physical Model. The spanwise and normal dimensions of the rectangular channel used in this paper are $150 \mathrm{~mm}$ and $50 \mathrm{~mm}$ respectively. The channel extends periodicity and the minimum period field is given in Fig. 1. Dimple/protrusion which has a diameter of $75 \mathrm{~mm}$ and a depth of 15 is placed in the center of the bottom surface, shown in Fig. 2.

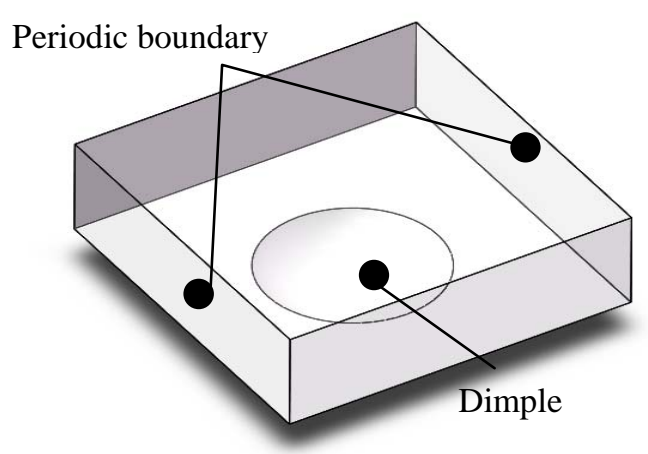

Fig. 1 Minimum period domain of channel

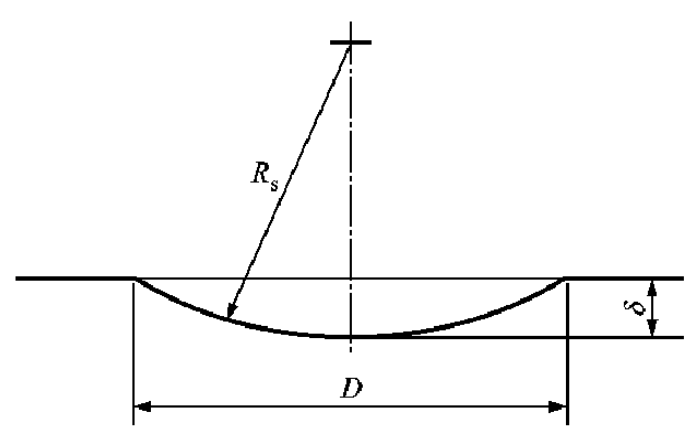

Fig. 2 Dimple cross-sectional geometry

Boundary conditions are defined as follows.

(1) Inlet and outlet periodic boundary.

(2) Inlet: temperature $\mathrm{T}=300 \mathrm{~K}$, pressure $\mathrm{P}=0.101 \mathrm{MPa}$, mass flow rate is determined by the $R e$ of 90000.

(3) Side walls: constant heat flux $q=1500 \mathrm{~W} / \mathrm{m}^{2}$.

Numerical Method. Supercritical carbon dioxide and air are set as the working fluid respectively. We solve the energy equation and Navier-stokes equations with the fluent solver, and details are set as follows.

(1) Turbulence model: realizable $k-\varepsilon$ model.

(2) Pressure-Velocity Coupling scheme: SIMPLEC.

(3) Spatial Discretization: Second Order.

Parameter Defination. The hydraulic diameter is defined as

$$
D_{h}=\frac{4 A}{P}
$$

where $P$ is the wet week. The heat transfer $\mathrm{Nu}$ is

$$
N u=\frac{h D_{h}}{\lambda}
$$

where $h$ is the heat transfer coefficient. The flow resistance coefficient $f$ is

$$
f=-\frac{(\Delta p / L) D_{h}}{2 \rho U_{m, i n}^{2}}
$$

where $\Delta p / L$ refers to the pressure drop per unit length in the flow direction. The comprehensive thermal characteristic $T P$ is

$$
\begin{aligned}
& T P=\left(\frac{N u}{N u_{0}}\right)\left(\frac{f}{f_{0}}\right)^{-\frac{1}{3}} \\
& N u_{0}=0.023 \operatorname{Re}^{0.8} \operatorname{Pr}^{0.4} \\
& f_{0}=0.046 R e^{-0.2}
\end{aligned}
$$

\section{Results and Conclusion}

Flow Characteristics. It can be seen from Fig. 3 that fluid separates in the leading edge of dimple and attaches in the trailing edge. Symmetrical separation bubbles forms on both sides above the 
dimple surface. From the temperature contours in Fig. 3, it is obvious that the temperature of dimple leading edge is high while the temperature of trailing edge is low. For the protrusion cases, fluid crashes in the leading edge of protrusion and separates in the trailing edge with the forming of symmetrical separation bubbles. The flow characteristics lead to the temperature distribution which shows low temperature in the leading edge and high temperature in the trailing edge. It is also evident that supercritical carbon dioxide cases own larger low-temperature region and smaller high-temperature region than air cases.

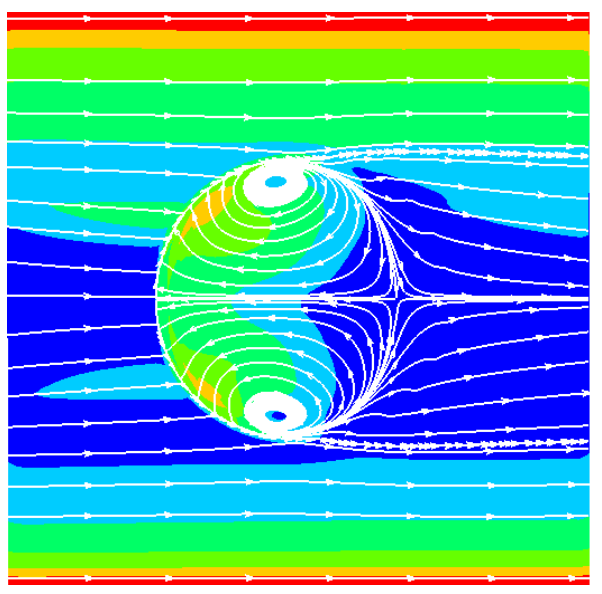

(a)

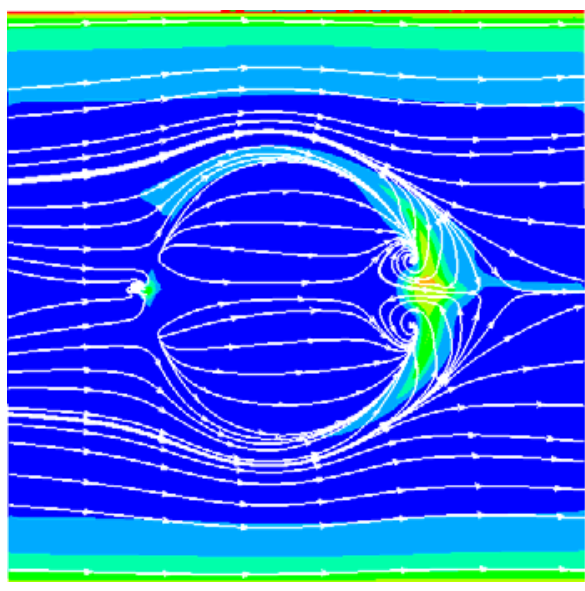

(c)

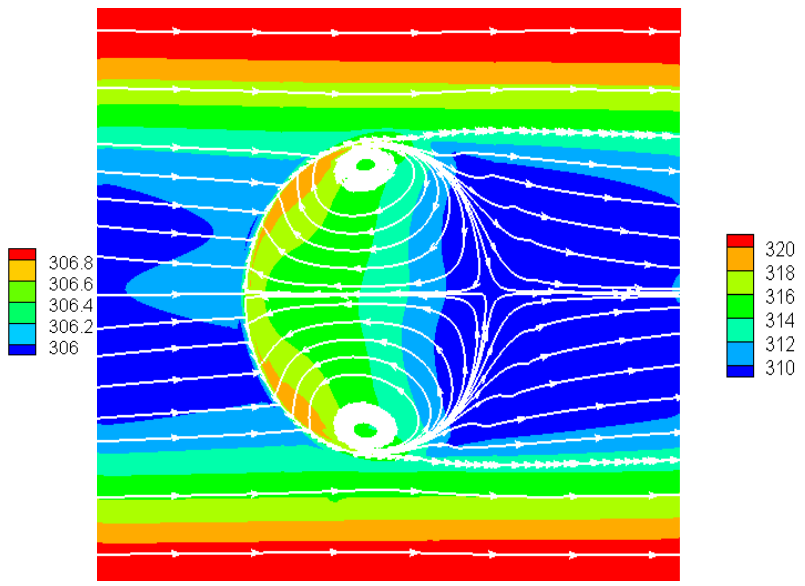

(b)

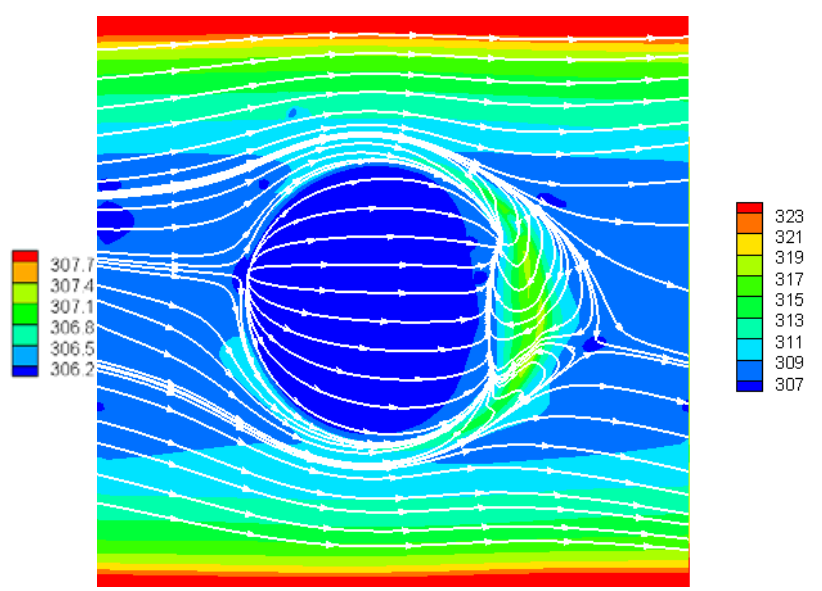

(d)

Fig. 3 Limiting stream lines and temperature contours of the dimple/protrusion cases: (a) dimple, supercritical carbon dioxide, (b) dimple, air, (c) protrusion, supercritical carbon dioxide, (d) protrusion, air

Heat Transfer and Pressure Drop Characteristics. The heat transfer coefficient $\mathrm{Nu}$, flow resistance coefficient $f$ and comprehensive thermal characteristic $T P$ of all calculated cases are listed in Table 1.

Table 1 Heat transfer, flow resistance and comprehensive thermal characteristics

\begin{tabular}{ccccc}
\hline Case & & $N u / N u_{0}$ & $f / f_{0}$ & $T P$ \\
\hline \multirow{3}{*}{ Supercritical carbon dioxide } & Flat & 2.76 & 1.34 & Ref \\
\cline { 2 - 5 } & Dimple & 2.89 & 1.77 & 2.39 \\
\cline { 2 - 5 } & Protrusion & 3.11 & 2.34 & 2.34 \\
\hline \multirow{2}{*}{ Air } & Flat & 1.06 & 1.4 & Ref \\
\cline { 2 - 5 } & Dimple & 1.19 & 1.84 & 0.97 \\
\cline { 2 - 5 } & Protrusion & 1.32 & 2.2 & 1.02 \\
\hline
\end{tabular}


For Table 1, we can find:

(1) For the same working fluid, the heat transfer $\mathrm{Nu} / \mathrm{Nu}_{0}$ of protrusion case is higher than that of dimple case following the flat case and flow resistance $f / f_{0}$ presents the same pattern.

(2) The $\mathrm{Nu} / \mathrm{Nu}_{0}$ of supercritical carbon dioxide cases is far higher than the $\mathrm{Nu} / \mathrm{Nu}_{0}$ of air cases while supercritical carbon dioxide cases present the similar flow resistance $f / f_{0}$ with air cases. The comprehensive thermal characteristic $T P$ of supercritical carbon dioxide cases is about 2.3 times that of air cases.

(3) For the supercritical carbon dioxide cases, dimple case presents better comprehensive thermal characteristic than protrusion case.

\section{References}

[1] BELEN'KIY M, Gotovskiy M A, Lekakh B M, et al. Heat Transfer Research, 1993, 25(2): 196-203.

[2] Moon H K, O’Connell T, Glezer B. Journal of Engineering for Gas Turbines and Power, 2000, 122(2): 307-313.

[3] Ligrani P M, Mahmood G I, Harrison J L, et al. International Journal of Heat and Mass Transfer, 2001, 44(23): 4413-4425.

[4] Jibing LAN, Yonghui XIE, Di ZHANG. Journal of Xi'an Jiaotong University, 2011, 45(7): 89-94.(In Chinese)

[5] Zhongyang Shen, Yonghui Xie, Di Zhang. Journal of Xi'an Jiaotong University, 2013, 47(3): 108-113.(In Chinese) 